Historic, Archive Document

Do not assume content reflects current scientific knowledge, policies, or practices. 
LEGRON FLORAL CO.

W. R. LEGRON, Prop.

125 Amherst Drive, - Toledo, Ohio

\section{Wholesale Gladiolus Price List}

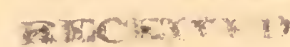

JAN 17 :

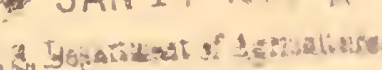

SETPPING TIME Wie send bulbs out all through Winter Winter months but do not start them during zero weather.
When temperature runs around 20 above, we are saie to mail. Bulbs can not be insured against freezing by mail. Express companies insure against freezing without extra charge. We do not lose over two paekages per year by mail from freezing the way we pack.

GUARANTEE. If any of cur bulbs are not satisfactory when received, return them and we will refund your money with charges both ways.

MINIMUM orders are one dollar. 25 per cent will hold your order until April first, 19.31, when they will be sont C. O. D. No orders are sent C. O. D. unless 25 per cent has been paid. This applies to TELEGRAMS as well. The 25 per cent can be telegraphed.

PREPAII). All orders amounting to $\$ 2.00$ or more will be sent out Prepaid. Less than $\$ 2.00$ will be scut posige collcet. Less than $\$ 1.00$ orders can not be filled.

SIZES. "L" at top of a column of prices mcans LARGE and this in turn means bulbs $11 / 4$ inches in diameter u1 " $M$ ". MEDIUM, $3 / 4$ inch to $1 \frac{1}{4}$ inch. " $S$ ", SMALL, all bulbs under $3 / 4$ inch.
PRICES. The minimum amount that we can afford to sell any item in this list is TEN CENTS. Make your item figurc 10c or more. For example, Adriatic is listed in Large at $1 \mathrm{c}$ cach. Order 3 bulbs at $4 \mathrm{c}$ each which makes $12 \mathrm{c}$ in one sack. Medinm is listed at $2 \mathrm{c}$ each. Order 5 bulls at $2 \mathrm{c}$ which makes $10 \mathrm{c}$ in one sack. Small are listed at lc cach. Orcicr 10 bulbs which makes $10 \mathrm{c}$ in one sack. On next page A. B. Kunderd is listed in Large at 8c cach Orcler 2 bulbs niaking $16 \mathrm{c}$ in one sack. If for any reason yout want only 1 bulb we will sell it to you for $10 \mathrm{c}$ where it is listed less than $10 \mathrm{c}$

********* Whon the * appears before the price it indicates that wc have this size in quantities and will allow you $25 \%$ dicount in lots of 25 to 100 . Do not take this discount on items not marked with * as it delays your order while we write you and await your reply.

BLLBLETS are only sold in quantities as listed as these arc counted and put in sacks before the Selling scason starts.

DISE $\Lambda$ SES. Bulbs were all treated chemically before planting and have been planted on virgin sandy soil each year for the past seven years.

\section{VARIETY}

ADRIATIC

ATPANIA

ALILAIIERA

ANTHEIVI

ARCOLA

ATHERTON

ATREUS

AUTULV:

AVALON

POPOLINK

PRHAK O'DAY

PUPELES

PUT DLEDEE

THAS IOHIER

CHERRI KING

CRES QUEEN

CRINISON GLOW

DEFIANCE

DIENERS \#183

" AM.PEAUTY

" THITE

D J WHITNEY

DORIS

DRA. GIORIOSO

DR COTHRAN

DR JAChSOIN

DUIHI APS

RPONY

E T SHAYLOR

ELF

FT.IZ.TAEOR

E KIRIIJAND

EXQUISITE

FAIRFAX

F J SYIvIVES

FONTAINE

GLINDATE

GOLD

GOLIEN KING

" SWATIOW

GRETCITN TANG

HAWAII

HHLGA

\section{IV S}

$4 c * 2 c * 1 c$ IE⿰氵工丁口iN TODD

*4c*2c $1 \mathrm{c}$ HIUTILID LAD$2 \mathrm{c}$ INDIHI

$4 \mathrm{c} 2 \mathrm{c} 1 \mathrm{c}$ JACH. TOIDON

*40*2c J TOR IIS.TRIN

7c 2c 1c JEWLL

1c $2 \mathrm{C} 1 \mathrm{c}$ JOE COLEl hil

4c JULA

tc 2c 1 c JUilizo

$* 4 c * c c^{*} 1 \mathrm{c}$ KART VOTKLRT

* $4 \mathrm{c} 2 \mathrm{c}$ LEOTA

$4 c 201 \mathrm{C}$ LILIIAN WLI

2c 1 e IIIYY LER Al?

2c 1o LILI WHIEE

*2c LORETIE

*2c*1c I,OUISE

$4 c 2 c 1 c$ IIATDNTS 1TT USSH

*4c*2c*1c IY AJESTIO

2c $1 \mathrm{c}$ IIARIE KUNDERO

* $4 c * 2 c$ IIITIJY

4c $2 \mathrm{C} 1 \mathrm{C}$ MING TOI

$* 4 \mathrm{c} * 2 \mathrm{c} * 1 \mathrm{c}$ HONO

*4c*2c IORINING GIORY

$* 4 c * 2 c * 1$ c IIRS DR NORTOI

*2c*1c IRS I PETERS

4c $2 \mathrm{c} 1 \mathrm{c}$ IIRS PENDIETON

*4c*2c I:RS II DOTHIN

2c 1 c IURS J R WALSH

$* 4 c * 2 c * 1 c$ I/RS SCFVHPPE

$4 c$ 2c 1o lRS l. S EURK $4 c * 2 c * 1 c$ IRS LOFRI MN

$4 c * 2 c * 1 c$ NRS S IIJSJ.

$4 \mathrm{c} 2 \mathrm{c} 1 \mathrm{C}$ l.RS W W.IIT * 2c*1c M T.S R.THARTSON

$4 \mathrm{c} 2 \mathrm{c} 1 \mathrm{c}$ lin IRd

2c 10 MISTIS

*2c*1c IAHAB

1c $2 \mathrm{c} 1 \mathrm{c}$ INAUTLIUS

$* 4 \mathrm{c} * 2 \mathrm{c} * 1 \mathrm{c}$ NIEOG A

*2c*1c NIAGAR̃A

* $2 \mathrm{c}^{*} 1 \mathrm{c}$ NOXaTí

$4 c * 2 c * 1 c$ PF,ACE

$* 1 \cdot \cdot \times 2 \mathrm{c}^{-\times 1} \mathrm{c}$
I. I. S

Is I. S

*20*1 e PINR IIIII

$4 \mathrm{c} 2 \mathrm{c}$

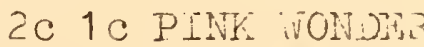

$4 c * 2 c * 1 c$ PRES HOORE

$4 \mathrm{c} * 2 \mathrm{c} * 1 \mathrm{c}$ PR OA WHLAS

CC 1 C UUINTON

*4c*2c RHIITI

$4 c 2 c$ RED CLOUD

*4c*2c RD COPPER

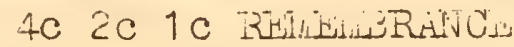

2c 1 c ROIIAIJCL

2c ROSE ASH

*4c*2c*1c ROSELTA

*4c*2c BOSELIRI

*4c*2c*1c ZOSE PRECOSE

*2c*1c ROUTi MORCH

*2c SARIH LILIE

*4c*2c*1c SChuta

* $4 \mathrm{c} * 2 \mathrm{c}$ SCIR PRITCEPS

4c $2 \mathrm{c} 1 \mathrm{c}$ SuIita

$* 10 * 2 c$

2c $1 \mathrm{c}$

$4 \mathrm{c} 2 \mathrm{c}$

$* 1 c * 2 c * 1 c$

$2 \mathrm{c} 1 \mathrm{c}$

$* 1 c * 2 c * 1 c$

$2 \mathrm{c} 1 \mathrm{c}$

$* 4 \mathrm{c} 20$

$* 4 c * 2 c * 1 c$

$* 4 \mathrm{c} * 2 \mathrm{c} * 1 \mathrm{c}$

$* 2 \mathrm{c} * 1 \mathrm{c}$

$* 2 c * 1 \mathrm{c}$

$* 4 c * 2 c * 1 c$

$4 \mathrm{c} 2 \mathrm{c}$

$* 4 c * 2 c$

$* 4 c * 2 c$

$4 c * 2 c * 1 c$

$4 \mathrm{c} 2 \mathrm{C} 1 \mathrm{c}$

4c $2 \mathrm{c} 1 \mathrm{c}$ SHEITA SISTER * $1 \mathrm{c} * 2 \mathrm{c}$

* $1 c * 2 c * 10$ SITRRA 4c 2c 1c

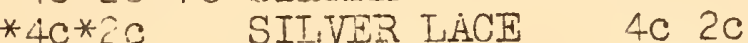

$4 \mathrm{c} 2 \mathrm{c} 1 \mathrm{c}$

$4 \mathrm{c} 2 \mathrm{c}$

$4 \mathrm{c} 2 \mathrm{c} 1 \mathrm{c}$

$2 \mathrm{c} 1 \mathrm{c}$

$2 \mathrm{c} 1 \mathrm{c}$

$4 c 2 c$

*4c*2c SUPERPA

2c 1c SURPRISE

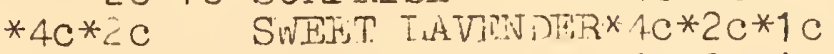

2c $1 \mathrm{c}$ THEDA $* 4 \mathrm{c} * 2 \mathrm{c} * 1 \mathrm{c}$

$2 \mathrm{C}$ TYCO ZATG $* 2 \mathrm{c} * 1 \mathrm{c}$

$4 c * 2 c * 1 c$ VAIITI

* $\mathrm{C}$ TISEA TILIET $2 \mathrm{c}$

*20 V_OTET PEAUTI

$* 40 * 20 * 1 \mathrm{c}$ WIITIKI $4 \mathrm{c} 2 \mathrm{c}$

$* 4 c * 2 c * 1 \mathrm{c}$ WIITE DIIIGHT 4c $2 \mathrm{c}$

*4c*2c WIIPRIIV

$4 \mathrm{c} 2 \mathrm{c}$ WIVE $D R O P$ 4c*2c*1c

*4c*2c*1c WISTARIA *4c*2c

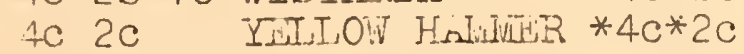

$4 \mathrm{C} 2 \mathrm{C} 1 \mathrm{c} "$ TREASURE $* 2 \mathrm{C} 10$

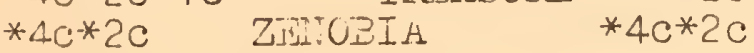





\section{VÁRIFIY}

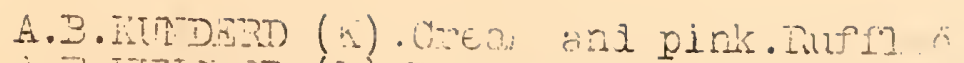

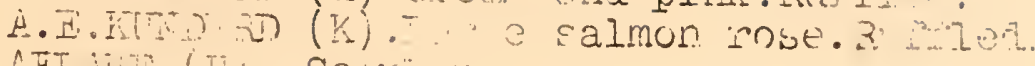

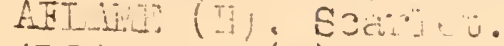

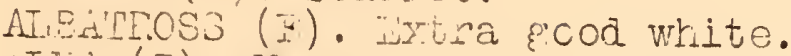

ATWA (D). Hernose piny. Ruffied.

ANDA PEITCHF (

ANNE THOMISCN (n). PaIE S liphur veliow.

MLRTICI GIOW (PAIrror). Apicot shade.

AREJTLUS ( $;$;. A Arutus pink.

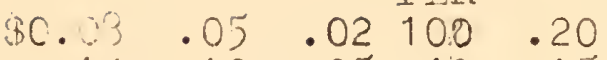

$\begin{array}{lllll}.1 \div & .12 & .05 & 10 & .15 \\ .05 & 15 & 10 & .15\end{array}$

ANTIONY ZONKER ( $K_{n}$ ). Deep sa? rnon rose. Diotches.

.750 .10

.1 .50

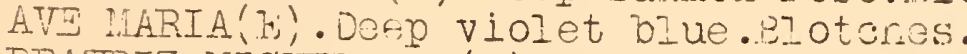

PEATRIZ MICEDTINÁ (D) . Jarge fieirt orange.

PLIVIDERE (D) .I.Lght salmon.

. .050

.12

.10

EENAAL TI(xIR (Piatt). Erilliant notsica rez.

.10

.10

BERNARD SHAW (D).Lilac, phI ox parie, and leinon.

EERIY SNOT (Scoulanx). Iavencier.

BETTY TOY (D). Creamy Wit, and pink.

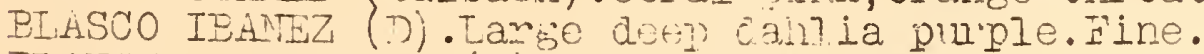

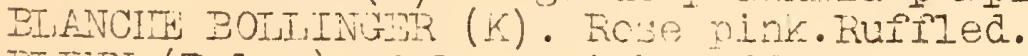

PIEiN (Eales). Saznon pirik. Trifoted.

EIUE ISLE ( $K$ ) . Tilac D Due with rea blotches.

PIUE I'ORCII (I:). Isight blie.

BCEDI (K) . Targe deep rose. Axtra rine.

PURBAMT'S INIXED. (Purbank). ASs onted colors.

CAIIFORNIA GIANT (D). Iallow a jie.

CAPT' COURACEOUS ( EIIIS). Large mottied red.

CAP' BOYNTON (Bo,nton). Iarge lavenü. Coo .

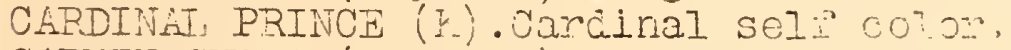

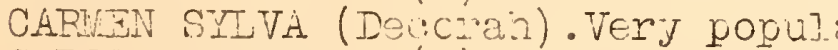

CAROLINE ESEERG (j). Flame scarlet

CAROLUS CLUSITS (II). Good commercial pint.

CARUSO (E). EAr crange red.

CATHERINE COI ENAN (C). Targe salnon pink.

CAITIAIA (D). Amararth pink. Yellow center.

CHAII Very Inrge rufr'tec pink.

CHARTEMAGN ( $\mathrm{H})$. Sunrise red.creamy blotch.

CHAS.F.FAIRDANKS $(K)$. Extra good red.

CHICAGO (K). Heavily ruifecd orange red.

COI. IINDEERGH (I) . Rea with white throat.

CORYPTEE (E). Extra fine pink.

CORONADO (Briges)-Large milk thita.Purple blotch."

COPPER PRONZE (K) . Hottled copper brorze. Fine. .08

CRINKIES (K) .Peach blosson pirk. Intensejy ruitiled.09, DETMONTE (D) . Aster purple.

DEI ORO (Durbank). Mía color bure selrmon.

DIXIE (K). Alriost black reã.

DORCAS ALIRICH(Dusinberre). Clowr rose, smoky tins..15

DORO (Puricanli). Cherry red on virite Eround.

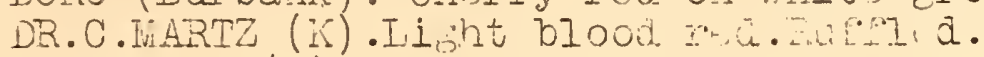

.10 .60

$.20 \quad .10$

.27 .04

.10 .06

$.30 \quad .20$

. 60

.20

.09
.07

.10
3.502 .50

$.10 .08 \quad .06$

.10

$\begin{array}{lllll}.09 & .06 & .03 & 100 & .20\end{array}$

$.05 \quad .03 \quad .02$

$.15 \quad .10$

1.00 .50

$* .06 * .04 * .02$

$25 \cdot 20$

DR. ELKINS $(K)$. White and lilas rlue.

DR.F.E.PENTE N (D).Deep peach zea.Jery popula. :

DR.InOODY(Kinjon). Parly lúvender.

. On

$\therefore \quad * 02 \quad 100 \quad .10$

10.07

1001.00

. 13,02

.02

$100 \cdot 30$

.10 .06

$.15 \quad .10$

$.10 \quad .07 \quad .03$

$10 \quad .15$

$* .08 * .05 * .03$

.60 .40

$.70 \cdot .50 \quad .25$

$.75 \cdot .35$

DR.NEISON SHOOY $(K)$. Targe tyrian rose. RuprIed. Extra.

DR. VhN FIEET(K) . Rose pina, Jelzum and cream.

Drefil $(K)$. Depo saluion rud.

DAVID STARR JORIAAT(D). Imnense flame colored.

DUCHESE OF YORK (Scotland). Clear ashy rose.

EARLY ELACK(IS). DEe ru ruby red.

EARTY PHIPPS (illis). Sinaiar but nior. earlier.

E.2.WIIIIATSON $\left(K_{2}\right)$. Solid mallow purple.

ELDIVA (D). Cerise, perciled crimson. A be dust.

ELFINA (Burbank). White, crimson and primise.

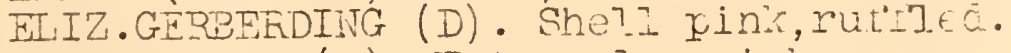

ETIZ. HARDEE (D). Waterme on pini.

EIIKHART $(\mathrm{K})$. Violet plirole.

$.35 \quad .25$

.05

.06

.08

.08 .05

100.10

$.03100 \quad .10$

$10 * 03$

$.10 .0 \%$

.100 .153

$100 \cdot 50$

$.00 \quad .04$

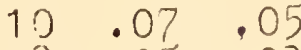

$\begin{array}{lllll}.03 & .03 & .03 & 100 & .25\end{array}$

$.12 .10 \quad .07 \quad 100 \quad .35$

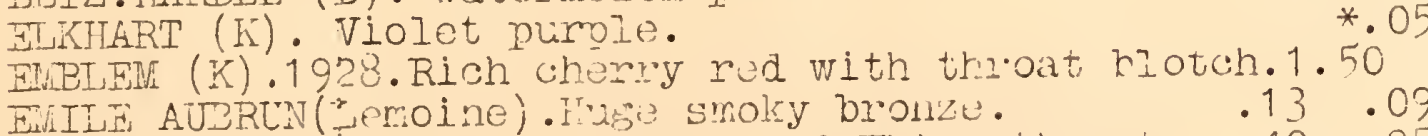

$10 * .07 * .05$

$* .05 * .02$

EINSICN (Errey). Eright scarlet red. White throat. .40

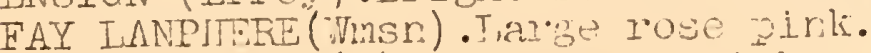

ESTHER OIISON (D). Sea ShoII pink.

ETHELYN (TISchor). Orenge TE I a ow.

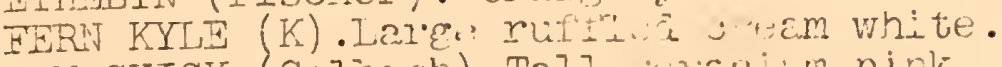

.10

.04

.04

$.02 * .04 * .02$

. $00 * .03$

.04

$.06 \quad .03$

$.05 * .03$

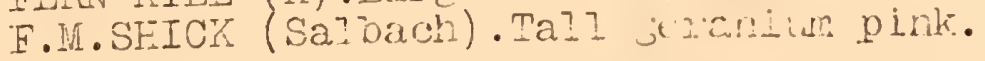

$.10 \quad .07 \quad .05$

$.0 ; \quad .03100 \quad .10$

$.06 * .04 * .02100 \quad .10$

$100 \cdot 20$

$100 \quad .20$

.10
.25

$25 \%$ Discount or all frices witr. * in Ious o 25. PRTPAT: 

FROST KING (EIIIso, Coral pink.

FULD' R RVORITE! $(K)$. Larse lavender rosa.

TAJ.,ANT LEAmBR $(A)$. Fxtra tall peach red.

$\$ 0.10 \quad .07$

$.05100 \quad 0.50$

GAY HJSBAR (Salbach). Irilliant oranse red.

GENAL KUROKI (KElWaY). Rosy red. Whl te throat. GEINVIVE BOTHIN(D).Pink and steel blue. Smoky.

\section{GTANT DIT}

.15 .03 GIANT NYIPH(C). A GIANT rose pink. axtra good cut.*.04*.02 GLADDIE POY (P). Grenadine sink.

GLADIS PLATH (D). Orchid with biotoh.

GLORIANA (Detscher).Pure salmon. No tarkings.

GOLD FAGTE (A). Earliest pure yeliton. Eufrled.

GOLDEN RROWN $(K)$. Mahosany red.

GOLDEN DREANI (Groff). Fine deep Jellan.

$.08 \quad .05$

.05
.04
.06

.02

$100 \quad .10$

.10 .06

*.06*.04*.02 $100 \quad .10$

.75

GOTDN TRTTS $(K)$. Ruffied yel .12 GOLDEN GLORY $(K)$. Ruffled rellow.

GOLDEN VEASURE (Kelway). JerY lar

.10

.08

.03

GOT DEN

GOLDEN SAIHON $(K)$. ROIOF same as name.

GOLDEN SNAPDRAGON $(i)$ 1929. INew typa.

HAREINGER (Sanford). Orange and red. Ruffled.

.25

FEART OF JUNIATA (D). Deep cherry searlet.

FEAVENLY BLUE (E). Near clear b? ue.

HENRY FORD (D). Rhodamine purple.

HEL WN HOWARD (A). Pronzy buff. Amber throat.

HELEN PHIPPS (D). Rose pink.

HELEN WIILS (SaIbach). Tall white, lemon tirroat.

.20

.08

.15

.08

.05

$.22 .18 \quad .12$

$.08 * .04 * .02$

$.05100 \quad .15$

.02

.05

$.10 \quad 10 \quad .25$

$100: 20$

HERDSTZAJPER (E). Very large orange salmon.

.10

.45

HINEMOA (Austral tallest glad. 0la rose. Sream thr..10

INDIAN CHI

1.75

INDIANAPOLIS $(K)$. Deep red. Purplish cast. Jar je.

INDIAN SUINER (K). Lavender rose pink. Ruffled.

IWA (Detscher). Pink with dark throat. Good.

J.A.CARPONE (D). Iridescent orange salmon.

J.D.OLIVER $(K)$. Lavender rose.

J.O.ARiv OUR (K) Coral salmon red. Ruriled.

JOERG'S WHITE (JOERG). Nothing .08

JOHN T.PIRIE (K) . Iviahogaby biown. Very popular.

JUBILEE (Kemp). Large lavender.

KATHARINE HESS (D). Geranium pink.

KING GEORGE (Scotiand). Dark red, creari blotch.

KING OF REDS $(K)$. Giant fiery crilison scarlet.

.25

.08

.07

.05

$50 \quad .50$

100.10

100.25

. 25

.00 .01

$.04 \quad .02$

$100 \cdot 50$

101.00

.10

$.06 *$.

.15

$* .04$
.10

.05

.03 .06

.06
.06

1.501 .00

.75

.60

KING SMOKe $(K)$.Kunderd's smoke. One of the best.

KING TUT (Davis). Salmon, narked deeper.

KUNDERD'S YEILON WONDER $(K)$. ButtercuO ye17.W.

1.25

LA COURONNE (Lemoine). White.

LA GAIETY (E). Light pink. Cherry blotch.

LA PAIOMA(Dusin). Bright orange. Extra good.

IAAVENDER PRIDE $(K)$. Smoxy lavenacr.

LA VERNE(Houdyshel). Straw yel l om and rlesh.

IONDON SNOKE(D) . Extra large gooa snoke.

LONGFELIOW (Decorah). Clear Lafrance pink.

IOIALTY (A). Tall fine yellow.

LJITAPY (K) . Orang', y I I ow and red.

IJTHER BUREANK (Purbink). Cherry pink.

IVANOTH WHITE (U D CO).Extra large white.

IIARIORA (Australia). Rich smoky iavender.

IARNIA(Kemp). Golden orange pink.

BARI FREY (Gelser).Silvery pink and lavender.

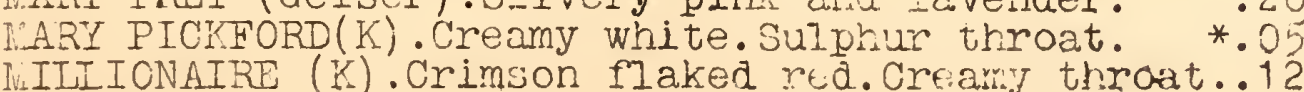

ININUET (C). Counted best Glad in WOBLD.I.avender. .14

IVOTHER NACHREE (Stevens). Lavendor and salmor pink.

I. RS.A. MEEKER(K) . American Peauty rose.

l. RS CAIVIIJ COOLIDGE ( $\mathrm{s}$ ).Pink with yellow throat. .25

IRS F.C. HORIDERGER (H). WH_TE.

IIRS.IJEN DOUGLAS(D). Desonia rose. Extra large. Good.09

Ui.ZS.P.W.SISSON (C). Creanj Iight pink.

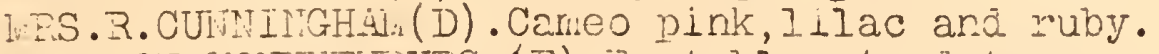

I. ES. VON KONINE2TURG (I) . Best biue to date.

IANSI FANKS(SaI) Apricot anc orange pink.

.75

.15

.15

.10

$\begin{array}{llll}.50 & .25 & 10 & .50\end{array}$

.10

.08

$100 \cdot 10$

.90

$.40 \quad .25$

$.08 \quad .05$

.10 .06

08

60.40

$10 \quad .15$

1001.50

101.00

.03

.03100

$100 \quad .20$

.

$.15 \quad .10$

$10 \quad .15$

1.75

$\begin{array}{lllll}30 & .20 & .15 & 10 & .20\end{array}$

*.03*.02 $100 \quad .10$

$* .03$

100

.10

.10

.08

.03

.02

.20

.68

.07

.05

10

.25

.05100 .10

$.05 \quad 100 \quad .15$

$.08 \quad .05$

$.17 \quad .12$

.05

$50 \quad .20$

$25 \%$ Discount on all jui tes wit. * in lutis of $2 \%$ 



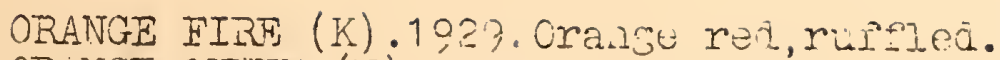
ORANGE QUESIN (E) . COZper orange.

PARAFOUNT $(K)$. Pin and cream.

PASADENA (D). Turisisir red. Iridescen.

PATL PFITIER. ( 9 ) Velvet purple.

PAUIINE KJNIRD (K). Rose piris. White triroat.

Peari of CAIFORIIA (kinssiey). Iight pink.

PERTA (Burbank). Cheiry cimsor.

PERŚA (ivat'l). Almost pipe klacis.

PFITTHR'S TRI TUUT (T) .09

PHINII (D) Jameo pink, blotched nirple.

PHAEINHON (E). Salmon pink and yEllow.

PHYLOS (D). Silvery pink.

FRINCESS OF ORANGE (K). Verrilinn rea.

PURPLE GLORY (K). Dest ruPfled jurplo.

PURPLE QUIFN (K) Deep violet purcie.RLfilec.

RAVENNA CREAM (A). Wiaze Tel lo\%, huff ard pirk. PED TORNAJO (EI? iso. Jirili icnt rea.

RED, WHITE AND GOID. (K) . CJ cr: as named.

RED (K) .1929. Dark ruby. RufII@1.

RICHARD DINITER (D) . Geranium rink.

RITA PECK (Tischer). Shell iris. Decurlet Iines.

R.J.KUNDERD (K). Deep vermLI ion.

ROSE MIST (Fischer). Rose rec, boratered buff.

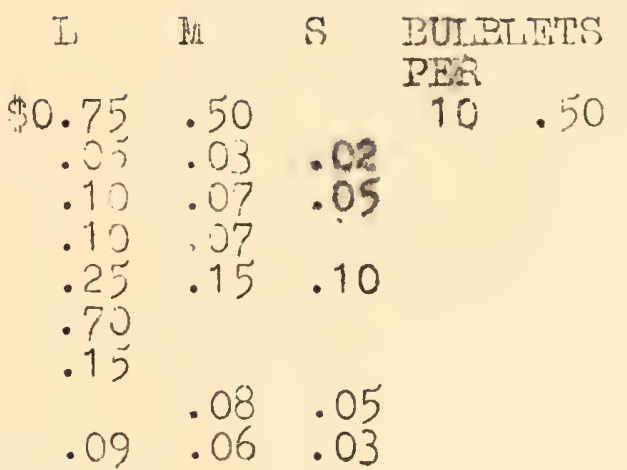

ROSI IVUIBERRI ( $i)$. ROse Aish colon.

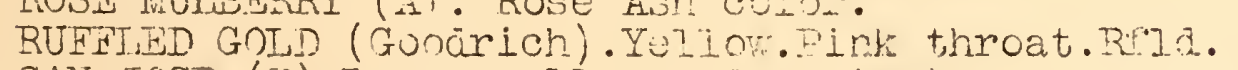

SAN JOEE (D) .IEnon JEIION alla arricot.

SCARTET (Burbank). Samon scantut.

STARDRIGH (Pales). Y 7 cw with red stripo.

STUTGARDIA (E). Jrilizicht onange.

SUNM (Eurbank) Shsmry red, flaked minon.

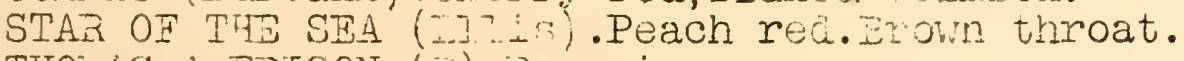
THOHAS A. IDISON (j) .

THAIS VALDIFAR (D). InIdescert olc roso.

TEE IMIR (Pcty). Juil sronze, shaled lighter.

THE PRESIDENT ( $K$ ) . Litie a rea canna.

IIGER (K). Smoky bìue anō salmon ruc.

TIGER FAUE (Eurvank). r] lishod rose.

IIPPECANOE (F) .192\%. Lavender. Rurfled.

VEILED ERIJIIANC (A). Pin tinted bluo.

VICTOR (EIII). Uruan YUlIOW.Vtry large. Rufflud.

TIOLET GTORY (K). Eich deE ViOlet. Ruffilcá.

THITE OEAHID (K). Whitc with red trroat.

.06

.10

*.0́ *.04

.25 .20

$.03 \quad .05$

.08 .05

.20 .10

.10
.25

.03

.50

$* .05 \quad .02$

$\begin{array}{lll}.05 & .03 \quad .07\end{array}$

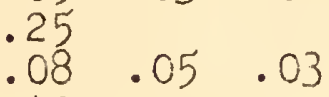

.10

.05

$100 \quad .10$

$.40 \quad .25$
$.08 \quad .05$

$* 10$

$.10 * 0$

$10 \quad .20$

W.H.PHIPPS (D).Lafrance pink ana sämon rose.

YEIION PEREECTION (E) . COOA JEIl OW.

MIXED. ḾOStIY KJNDEED'S and YIENTZ'S. In full 100

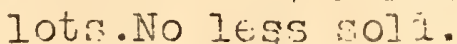

$.7 * .04$

$\begin{array}{llll}.10 & .05 & 25 & .50\end{array}$

$.08 \quad .04 \quad .02$

.40

.20

10.40

$.10 \quad .05 \quad .02$

.12 .06

$.08 \quad .04 \quad .02$

$\begin{array}{lll}.18 & .12 & .07\end{array}$

$.20 \quad .10 \quad .05$

.10 .06

.07

$* .05 * .03$

$.08 \quad .04$

.60

1.00 * $155^{\circ} .50$

A.ll above prices are prepaił if your order to $1 \mathrm{~s} \$ 2.00$ ol more.

$25 \%$ Discount on prices with * in lots of 25 wr mole.

orders for less than $\$ 2.00$ are sent postage ornut.

IRIS and PIONI LISTS will be read about WAY 1.21931.

IJE GRON FLORAT, CO

125 AIITISET DEIV

TOTEDO OHIO 
